\title{
4.2.3. Class II Microcins
}

\author{
Gaëlle Vassiliadis ${ }^{1, a}$, Delphine Destoumieux-Garzón ${ }^{2}$, and Jean Peduzzi ${ }^{{ }^{*}}$
}

\author{
${ }^{1}$ CNRS, Muséum National Histoire Naturelle, FRE3206, Case 54, 57 rue Cuvier, 75005 Paris, France \\ 2 CNRS, Ifremer, IRD, Université Montpellier 2, UMR5119, Laboratoire Ecosystèmes Lagunaires. Place Eugène \\ Bataillon, CC80, 34095 Montpellier cedex 5, France \\ a present address: Institut National de la Recherche Agronomique, Institut Micalis, UMR1319, Domaine de \\ Vilvert, 78352 Jouy-en-Josas, France \\ *: Corresponding author : Jean Peduzzi, Tel. 331407938 18. Fax: 33140793135. \\ email : peduzzi@mnhn.fr
}

\begin{abstract}
:
Class II microcins are 4.9 to $8.9 \mathrm{kDa}$ polypeptides produced by and active against enterobacteria. They are classified into two subfamilies according to their structure and their gene cluster arrangement. While class Ila microcins undergo no post-translational modification, class Ilb microcins show a conserved C-terminal sequence that carries a salmochelin-like siderophore motif as a posttranslational modification. Aside from this C-terminal end, which is the signature of class Illb microcins, some sequence similarities can be observed within and between class II subclasses, suggesting the existence of common ancestors. Their mechanisms of action are still under investigations, but several class II microcins use inner membrane proteins as cellular targets, and some of them are membraneactive. Like group B colicins, many if not all class II microcins are TonBand energy-dependent and use catecholate siderophore receptors for recognition/translocation across the outer membrane. In that context, class Ilb microcins are considered to have developed molecular mimicry to increase their affinity for their outer membrane receptors through their salmochelin-like post-translational modification.
\end{abstract}




\subsubsection{Introduction}

Class II microcins are higher molecular mass microcins $(4.9$ to $8.9 \mathrm{kDa})$ and their peptide backbone does not undergo extensive post-translational modifications (Duquesne et al., 2007). It has been recently shown that class II microcins were markers of virulence patterns in uropathogenic Escherichia coli (Azpiroz, Poey and Laviña, 2009). In this review, we have successively detailed the structures, the genetics, the biosynthesis, and the mechanisms of action of class II microcins. The organization of class II microcin gene clusters involves at least four clustered genes organized into one single or several transcription units. The minimal structure is composed of (i) the gene encoding the microcin precursor, (ii) an adjacent self-immunity gene, which encodes the self-immunity protein that protects the producing strain from its own antibacterial substance, and (iii) at least two genes encoding an inner membrane ABC (ATP-binding cassette) transporter and its accessory protein responsible for the secretion of the microcin. The nonspecialists should be aware that the name given to each gene is not standardized throughout the different microcin gene clusters. While class Ila microcins are not post-translationally modified, class Ilb microcins show a conserved C-terminal sequence that carries a salmochelin-like siderophore motif as a post-translational modification. They are therefore considered as antibacterial siderophore-peptides (Thomas et al., 2004). Siderophores are small iron chelators synthesized by bacteria and fungi for the uptake of the very low amount of iron available in physiological media (Andrews, Robinson and Rodriguez-Quinones, 2003). The enzymatic pathways leading to the class Ilb microcin post-translational modification have been recently studied (Nolan et al., 2007; Vassiliadis et al., 2007; Nolan and Walsh, 2008; Mercado et al., 2008) and various steps of class Ilb microcin biosynthesis could be achieved in vitro. The mechanisms of action of class II microcins are still under investigations, most of the knowledge being acquired on their recognition/uptake. Like group B colicins, many if not all class II microcins are TonB- and energydependent and use catecholate siderophore receptors for recognition/translocation across the outer membrane (Chehade and Braun, 1988; Trujillo, Rodríguez and Laviña, 2001; Patzer et al., 2003; Thomas et al., 2004; Strahsburger et al., 2005). As a consequence, the salmochelin-like posttranslational modification of class $\mathrm{Ilb}$ microcins increases their affinity for their outer membrane receptors (Thomas et al., 2004). Such a molecular mimicry is a unique case among antimicrobial peptides. 


\subsubsection{Class Ila microcins}

Microcins (Mcc) belonging to this subclass are characterized by the absence of posttranslational modification. MccV and MccL contain disulfide bond(s) whereas Mcc24 would be a linear unmodified peptide lacking such bond.

\subsection{Genetics and structure}

Class Ila microcin gene clusters (Fig. 1) are composed of only four plasmid-borne genes. MccV, formerly colicin V (ColV) (Fredericq, 1949) was the first antibiotic substance reported to be produced by E. coli (Gratia, 1925). MccV is secreted by various $E$. coli strains harbouring large (> 80 kb) and low copy number pColV plasmids (Waters and Crosa, 1991). MccL is produced by E. coli LR05 isolated from poultry intestine (Gaillard-Gendron et al., 2000), while Mcc24 (formerly colicin 24) is secreted by the uropathogenic E. coli 2424 and its genetic determinants are located on the $43.5-\mathrm{kb}$ conjugative plasmid p24-2 (O'Brien and Mahanty, 1994). MccV and MccL gene clusters are composed of four genes organized in two converging transcription units (Gilson, Mahanty and Kolter, 1987; Chehade and Braun, 1988; Gilson, Mahanty and Kolter, 1990; Pons et al., 2004). The genes cvaC and mclC, encoding a 103-amino acid MccV and a 105-amino acid MccL precursor, respectively, as well as the self-immunity genes $\mathrm{cvi}$ and $\mathrm{mcll}$ form the first operon. The second operon contains two genes ( $c v a A / m c / A$ and $c v a B / m c / B$ ) involved in the MccV and MccL export, respectively (Gilson, Mahanty and Kolter 1990; Pons et al., 2004). In MccL gene cluster, downstream of mcll, one open reading frame (ORF) exhibits $98 \%$ identity with cvi, which makes MccL-producing strains resistant to MccV (Sablé et al., 2003). Mcc24 gene cluster (GenBank accession number U47048) revealed four genes that would belong to one single operon (O'Brien and Mahanty, 1996, unpublished work). The genes $m t f S$ and $m t f l$ encode the putative 88-amino acid precursor and self-immunity protein of Mcc24, respectively, whereas $m t f A$ and $m t f B$ encode proteins similar to CvaA and CvaB, respectively.

The mature $\mathbf{M c c V}$ is a 88 -amino acid peptide, originating from a 103-amino acid precursor (CvaC) that possesses a single disulfide bond located in the C-terminal sequence (Table 1) (Håvarstein, Holo and Nes 1994). MccL, which is generated from a 105-amino acid precursor (McIC), is composed of 90 unmodified amino acids (Pons et al., 2004). MccL is an anionic and highly hydrophobic peptide characterized by two disulfide bonds (Pons et al., 2004). MccL and MccV share 
an identical 13-amino acid $C$-terminal sequence that contains one disulfide bond (Table 1). Mcc24 has neither been isolated nor characterized. Nevertheless, its precursor amino acid sequence, which shares homology with MceA, a class Ilb microcin precursor (Table 1), but lacks the 10-amino acid $C$ terminal sequence typical of class Ilb microcins (see 4.2.3.3.2), and its gene cluster, which contains four genes only, suggest strongly that Mcc24 belongs to class Ila microcin. The putative mature Mcc24 corresponds to a 73-amino acid peptide devoid of disulfide bond.

\subsection{Maturation and export}

Many bacterial proteins are translocated across the inner membrane by the Sec pathway (de Keyzer, van der Does and Driessen, 2003). Nevertheless, some bacteriocins, like class II microcins, use ATP-binding cassette (ABC) transporters (Davidson and Maloney, 2007; Hollenstein, Dawson and Locher 2007; Moussatova et al., 2008). For all class II microcins, maturation (cleavage of the leader peptide) and export appear to occur simultaneously. Except for MccV, whose mechanism of export has been partially characterized (Zhong, Kolter and Tai, 1996), the class II microcin export machineries have been mainly identified based on genetics and sequence identity analysis. The class II microcin export machinery displays a canonical structure consisting of three components.

The first component is an ABC transporter. Encoded by the microcin gene cluster, this protein is an homodimer carrying a nucleotide fixation domain. CvaB would be responsible for the export of MccV and, based on their highly similar sequence (up to $70 \%$ identity), MclB and MtfB, for the export of MccL and Mcc24, respectively. All three proteins are similar to ABC transporters responsible for the export of other antibacterials such as class II bacteriocins from Gram positive bacteria (Håvarstein, Diep and Nes 1995). Typically, ABC transporters exhibit three domains: (i) an Nterminal domain, supposed to be located in the cytoplasm (Franke et al., 1999; Wu et al., 2004), with a protease activity likely involved in the leader peptide cleavage during export, (ii) a central transmembrane domain, poorly conserved, and (iii) a C-terminal domain carrying a highly conserved nucleotide-binding cassette required for ATP binding (Benabdelhak et al., 2003). A model was proposed in which the binding of the MccV precursor to CvaB promotes a transition between an inactive dimer bound to nucleotide diphosphate to a high-energy dimer bound to nucleotide triphosphate (Guo, Harrison and Tai, 2006). 
The second component of the export machinery is the ABC transporter accessory protein. Based on bioinformatics studies, this protein, which is encoded by the microcin gene cluster, would be periplasmic with an $\mathrm{N}$-terminal transmembrane helix that serves as an anchor at the inner membrane (Skvirsky, Reginald and Shen, 1995; Franke et al., 1996). Like ABC transporters, accessory proteins are also highly conserved. CvaA, MclA and MtfA are accessory proteins for MccV, MccL, and Mcc24, respectively. Their function in microcin secretion remains unclear. However, they have been proposed to serve as a connector between the $A B C$ transporter and the third component of the class II microcin export machinery (Gilson, Mahanty and Kolter, 1990; Pons et al., 2004).

The third component of the machinery is TolC, located at the outer membrane. Unlike genes encoding the $A B C$ transporter and the accessory protein, tolC is located on the bacterial chromosome outside of the microcin gene cluster. This protein forms a trimeric channel with an a helix in the periplasmic space, and a $\beta$ barrel channel spanning the outer membrane (Koronakis et al., 2000; Koronakis, Eswaran and Hughes, 2004). The expression of TolC is required for the production of extracellular antimicrobials by MccV- and MccL-producing strains (Gilson, Mahanty and Kolter, 1990; Pons et al., 2004). Therefore, TolC is considered to be required for the export of class II microcins.

\subsection{Recognition/uptake and mechanism of action}

Class Ila microcins have been poorly studied in terms of mechanism of action compared to class IIb. Most of the data are speculative and based on sequence similarities with microcins with known mechanism of action.

MccV (ColV) is active against Gram-negative bacteria belonging to the genera Escherichia, Klebsiella, Salmonella and Shigella (Håvarstein, Holo and Nes, 1994). Like class Ilb microcins, MccV is recognized by an outer membrane receptor for catecholate siderophores, and its uptake is TonBdependent (Chehade and Braun, 1988). It is therefore likely taken up into sensitive cells at the expense of energy which is provided by the proton motive force of the inner membrane. However, while class Ilb microcins are recognized by FepA, Cir and Fiu, Cir alone appears to be involved in MccV uptake (Chehade and Braun 1988). MccV activity also depends on the inner membrane protein SdaC, also involved in serine uptake (Gerard, Pradel and Wu, 2005). Although the mechanistic role of $\mathrm{SdaC}$ in MccV uptake and/or activity remains unknown, it could drive MccV to the inner membrane, 
thereby promoting channel formation and subsequent disruption of membrane potential (Yang and Konisky,1984).

MccL spectrum of activity covers a large number of Gram-negative species including E. coli, Salmonella enterica, Klebsiella oxytoca, Shigella sonnei, Shigella flexneri, Pseudomonas aeruginosa, and Providencia stuartii (Pons et al., 2004). However, to date MccL has been poorly studied in terms of mechanism of action. Since it displays a 32-residue C-terminal sequence highly similar to that of $\operatorname{MccV}$ (87.5\% identity), it could also have membrane-permeabilizing properties (Pons et al., 2004). This conserved region could also be required for recognition and uptake, since like MccV, MccL is recognized by Cir and imported in a TonB-dependent manner (Sablé, personal communication).

Mcc24 was reported to be active against the enterobacteria E. coli and S. enterica Thyphimurium but not against Listeria monocytogenes or Campylobacter jejuni (Wooley, Gibbs ans Shotts Jr, 1999). To date, nothing is known regarding its uptake and mechanism of action. It is actually the only class II microcin for which nothing is known in terms of recognition by catecholate siderophore receptors and their associated TonB-ExbB-ExbD complex. Mcc24 displays striking sequence similarities with MccE492 (Lagos et al., 1999) but lacks the C-terminal region required for recognition by catecholate siderophore receptors. Based on their sequence identities, it was suggested that Mcc24 and MccE492 could have similar cellular targets and would both require ManYZ at the inner membrane (Bieler et al., 2006).

\subsubsection{Class Ilb microcins: the siderophore-microcins}

Class Ilb microcins are linear polypeptides carrying a $\boldsymbol{C}$-terminal siderophore as a posttranslational modification (MccE492, MccH47, MccM and presumably MccG492 and Mccl47). These microcins are characterized by a highly conserved 10 -amino acid $C$-terminal sequence, which is considered their signature (Vassiliadis et al., 2010).

\subsection{Genetics}

Contrary to class Ila microcins, which are all plasmid-encoded, class Ilb microcins are chromosome-encoded and their gene clusters show a complex transcriptional organization (Fig. 2). 
In addition to the four genes described in class Ila microcin gene clusters, genes encoding posttranslational modification enzymes are encountered in class Ilb microcin gene clusters.

The best characterized class Ilb microcin is MccE492, which is secreted by Klebsiella pneumoniae RYC492 (de Lorenzo, 1984). The entire MccE492 gene cluster is contained within a 13kb DNA fragment that has been cloned in E. coli (Wilkens et al., 1997). Ten genes (mceABCDEFGHIJ) (Lagos et al., 2001), organized at least in five transcription units, are necessary for MccE492 biosynthesis. The gene $m c e A$ encodes the 99 -amino acid MccE492 precursor and mceB is involved in the self-immunity towards MccE492 (Lagos, Villanueva and Monasterio, 1999). Other genes are required for MccE492 post-translational modification. Those are $m c e C$, mceD and mcel, which encode proteins homologous to a glycosyltransferase, an enterobactin esterase, and an acyltransferase, respectively, (Nolan et al., 2007; Vassiliadis et al., 2007), as well as mceJ, which does not display evident homologies with known genes (Corsini et al., 2002; Nolan et al., 2007). Two genes, $m c e G$ and $m c e H$, are necessary for the export of MccE492. They encode an ABC transporter and an accessory protein, respectively. The mceF gene would also be involved in export (Lagos et al., 2001) and the role of the last gene, mceE, remains unknown. The nucleotide sequence of this gene cluster has been recently corrected (GenBank accession number AF063590) showing that the orientation of mceFGHIJ is reverse to that previously described. The corrected sequence also unveiled six novel ORFs (mceS2/mceS3/mceM/mceL/mceX/mceK), among which mceL and mceM encode the 89-amino acid precursor of a new putative microcin termed MccG492 and its self-immunity protein, respectively (Vassiliadis et al., 2010).

As with K. pneumoniae RYC492, several microcins can be expressed by one single E. coli isolate. Thus, E. coli $\mathrm{H} 47$ was reported to express both MccH47 and Mccl47 (Laviña, Gaggero and Moreno, 1990; Poey, Aspiroz and Laviña, 2006). Similarly, E. coli Nissle 1917 (also named Mutaflor), E. coli CA46, and E. coli CA58 strains express MccM, MccH47 and possibly Mccl47 (Nissle 1925; Patzer et al., 2003; Duquesne et al., 2007; Vassiliadis et al., 2010). The genetic determinants required for $\mathrm{MccH} 47$, Mccl47 and MccM biosynthesis are all located within a 10.5-kb to 14-kb DNA fragment (Laviña, Gaggero and Moreno, 1990; Patzer et al., 2003; Vassiliadis et al., 2010). Analysis of the four microcin gene clusters showed that they share a common organization except for the 5 ' region located upstream of $m c h X$ (Fig. 2). Each microcin gene cluster is characterized by common genes (homologues) involved in microcin export. Those are $m c h E, m c h F$ and $m c m M$, the later being 
truncated in E coli H47 (Gaggero, Moreno and Laviña, 1993; Azpiroz, Rodríguez and Laviña, 2001; Patzer et al., 2003; Poey, Aspiroz and Laviña, 2006). Similarly, four genes required for the posttranslational modification of class Illb microcins are common to the microcin gene clusters. Those genes are $m c h A / m c m L, m c h S 1 / m c m K, m c h C$ and $m c h D$, which are homologous to mceC, mceD, mceJ and mcel from MccE492 gene cluster, respectively (Vassiliadis et al., 2010). However mcmL and $m c m K$ are lacking in E. coli Nissle 1917. Finally, two specific genes encode each microcin precursor and self-immunity protein. Thus, $m c h B$ encodes the 75-amino acid MccH47 precursor (Rodríguez, Gaggero and Laviña, 1999) and mchl confers the self-immunity towards MccH47 (Rodríguez and Laviña, 1998). The gene mchS2 encodes the 77-amino acid Mccl47 precursor and mchS3 confers the specific self-immunity towards Mccl47 (Poey, Aspiroz and Laviña, 2006). The $m c m A$ (formerly $m c m C$ ) (Braun et al., 2002), encodes the 92-amino acid MccM precursor, and $m c m l$ encodes the MccM self-immunity protein (Patzer et al., 2003). The role of mchS4 and mchX located between mchS3 and mchl remains unclear (Rodríguez and Laviña, 1998; Azpiroz and Laviña, 2004). In the $5^{\prime}$ region of microcin gene clusters from E. coli CA58 and Nissle 1917 are located genes encoding transposase and insertion sequences, which strongly supports the hypothesis of an horizontal gene transfer of microcin gene clusters. Complete or partial MccM gene cluster are also encountered in the pathogenicity island of the uropathogenic E. coli strains (Dobrindt et al., 2001; Welch et al., 2002; Dezfulian, Tremblay and Harel, 2004).

\subsection{Structures}

MccE492 was initially described as an 84-residue unmodified peptide (uMccE492) (Pons et al., 2002) deriving from the MccE492 precursor (MceA). Culture conditions were found to be critical to obtain mature MccE492, which carries an original post-translational modification as revealed by mass spectrometry and nuclear magnetic resonance (Thomas et al., 2004). This modification consists of a C-glucosylated linear trimer of $\mathrm{N}$-(2,3 dihydroxybenzoyl)-L-serine (DHBS) linked to the C-terminal serine carboxylate via an O-glycosidic bond (Fig. 3) (Thomas et al., 2004), a structure reminiscent of catecholate siderophores, and especially of salmochelin S4 (Hantke et al., 2003). Thus, MccE492 was the first example of a natural siderophore-peptide. With the characterization of novel microcins carrying a siderophore post-translational modification, the name of siderophore-microcins was given to class Ilb microcins. Indeed, MccM and MccH47 were recently shown to be 77 and 60 residue-peptides 
(Table 1) deriving from the microcin precursors $\mathrm{McmA}$ and $\mathrm{MchB}$, and carrying a C-terminal posttranslational modification similar to that previously described for MccE492 (Vassiliadis et al., 2010). As such, they unambiguously belong to the siderophore-microcin family. However, when isolated from $E$. coli Nissle 1917, MccM and MccH47 did not carry any modification. The absence of post-translational modification was correlated to the absence of $\mathrm{mcmL}$ and $m c m K$ in microcin gene clusters, two genes involved in the biosynthesis of the post-translational modification (Vassiliadis et al., 2010). Two other putative members, MccG492 and Mccl47, which remain to be fully characterized, would also belong to the siderophore-microcin family. After leader-peptide cleavage, putative MccG492 and Mccl47 would contain 74 and 62 residues deriving from the microcin precursors MceL and MchS2, respectively (Table 1). Consequently, the class Illb microcins form the first homogeneous family amongst microcins, the siderophore-microcins.

\subsection{Biosynthesis and export}

Several recent studies have shown that aside from the microcin gene clusters, genes involved in the biosynthesis of enterobactin and salmochelin are required for class Ilb microcin biosynthesis, and that both siderophores are precursors for the post-translational modification (Azpiroz and Laviña, 2004; Nolan et al., 2007; Vassiliadis et al., 2007; Mercado et al., 2008). This chapter describes the biosynthetic pathways that lead to siderophore-peptides, a unique combination of ribosomal and nonribosomal biosynthetic steps (Nolan and Walsh 2008).

\subsection{Enterobactin and salmochelin biosynthesis}

The maturation of class $\mathrm{Ilb}$ microcins is dependent on catecholate siderophores. Catecholatesiderophores are named according to the chemical group that chelates iron and are one class of siderophores among the 500 siderophore structures described until now (Neilands, 1995; Crosa and Walsh, 2002; Miethke and Marahiel 2007). Enterobactin (also termed enterochelin) is a catecholate siderophore that was first isolated in S. enterica Thyphimurium (Pollack and Neilands, 1970) and E. coli (O'Brien, Cox and Gibson, 1970), and later in Streptomyces (Fiedler et al., 2001), Klebsiella and Shigella species (Payne et al., 1983; Podschun, Fischer and Ullmann, 1992). It consists of a cyclic trimer of DHBS that chelates iron through its three dihydroxybenzoate (also termed catechol) groups. The coordination between enterobactin and iron is of the hexadentate type. Enterobactin was 
described as the best iron chelator isolated until now (Ecker, Matzanke and Kocher, 1986). More recently, a new class of siderophore, named salmochelins, was isolated from Salmonella enterica Thyphimurium (Hantke et al., 2003). Also produced by several uropathogenic $E$. coli and $K$. pneumoniae strains, salmochelins derive from enterobactin by the addition of one or two glucose moieties on the enterobactin catechol group.

\section{Enterobactin biosynthesis}

Most of the known siderophores are synthesized by the multienzymatic pathway and particularly by the nonribosomal peptide synthetases (NRPS) pathway (Miethke and Marahiel, 2007). Two main pathways lead to enterobactin biosynthesis.

The first pathway, which serves for the initial steps of enterobactin biosynthesis, is the shikimate pathway. From phosphoenol pyruvate and erythrose 4-phosphate, this pathway leads to chorismate, which is a common intermediate to enterobactin and aromatic amino acids biosynthesis. Genes involved in this pathway are aroABCDEFGHKL. Tyrosine, phenylalanine and tryptophan have been described as inhibitors of the shikimate pathway (Cobbett and Delbridge, 1987; Grove and Gunsalus, 1987; Heatwole and Somerville, 1992).

The second pathway, which serves for the last steps of enterobactin biosynthesis, involves an NRPS (Walsh et al., 1990; Crosa and Walsh, 2002; Raymond, Dertz and Kim, 2003; ). Extensive studies have been performed on specific NRPS which lead from chorismate to enterobactin. All genes involved in this pathway are located in the enterobactin gene cluster. This $20 \mathrm{~kb}$ fragment, organized in six operons, contains 16 genes. Seven (entABCDEF and $y b d B$ ) are involved in enterobactin synthesis, five (fepABCDG) in enterobactin uptake, one (entS) in enterobactin export, one (fes) in the release of iron from the siderophore, and two genes ( $y b d z$ and fepE) still have unknown functions. The enterobactin gene cluster is regulated by two fur (ferric uptake regulator) boxes enabling the transcription regulation according to bacterial iron concentration (Brickman, Ozenberger and Mclntosh, 1990). Thus, to achieve enterobactin biosynthesis, chorismate is subjected to three reactions leading to dihydroxybenzoate (DHB) (Sakaitani et al., 1990; Gehring, Mori and Walsh, 1998). Afterwards, DHB is activated by adenylation and charged onto an aryl carrier protein. In parallel, a serine is also activated by adenylation and charged onto a peptidyl carrier protein (Reichert, Sakaitani and Walsh, 1992; Lambalot et al., 1996; Gehring, Bradley and Walsh, 1997). DHB and serine are finally ligated by a condensation domain and transferred onto another peptidyl carrier protein. These steps are 
repeated three times and the final trimer of DHB-serine is transferred onto a thioesterification domain which cyclizes and releases simultaneously the trimer to finally produce the enterobactin (Shaw-Reid et al., 1999).

\section{Salmochelin biosynthesis}

Conversion of enterobactin into salmochelins requires a $10 \mathrm{~kb}$ DNA fragment containing five genes, iroBCDEN (Bäumler et al., 1996; Bäumler et al., 1998) and two fur boxes (Bäumler et al., 1996). The gene iro $B$ is involved in salmochelins biosynthesis, iro $C$ in salmochelins export, iroN in salmochelins import while iroD and iroE are involved in the release of iron chelated by salmochelins and degradation of these siderophores (Lin et al., 2005; Zhu et al., 2005). Enterobactin is diglucosylated by IroB on two catechol groups leading to salmochelin S4 formation (Bister et al., 2004; Fischbach et al., 2005). Salmochelin S2 is the linear form of salmochelin S4 while salmochelins S1, and SX are the monoglucosylated dimer and the monomer of DHBS, respectively. Salmochelins S2, S1 and SX are consequently degradation products of salmochelin S4.

\subsection{Siderophore microcin biosynthesis}

MccE492 was the first microcin found to carry a salmochelin-like siderophore as a posttranslational modification (Fig. 3). As a consequence, it has been the most extensively investigated of the siderophore-microcins. The genes $m c e C$ and $m c e D$ from MccE492 gene cluster display strong identities sequences with $i r O B$ and $i r o D$, which are involved in enterobactin glucosylation and linearization, respectively. In vivo and in vitro experiments showed that enterobatin and salmochelin are precursors for MccE492 post-translational modification (Vassiliadis et al., 2007; Mercado et al., 2008). Thus, while microcins use siderophore biosynthetic pathways for the initial steps of their posttranslational modification, the last steps are encoded by the microcin gene clusters themselves. It was recently shown for MccE492 that MceC and MceD monoglucosylate and linearize enterobactin, respectively (Nolan et al., 2007), and that Mcel and MceJ are responsible for the O-glucosydic bond formation between the modified enterobactin and the peptide backbone (Nolan and Walsh, 2008).

The biosynthesis of MccM and $\mathrm{MccH} 47$, the two recently characterized siderophore-microcins, was also investigated (Vassiliadis et al., 2010). Like MccE492, MccH47 post-translational modification is dependent on the enterobactin synthesis pathway (Azpiroz and Laviña, 2004). Interestingly, MchA and $\mathrm{McmL}$ display $85 \%$ identity with MceC, while MchS1 and McmK display $75 \%$ identity with MceD. 
By coupling gene complementation to mass spectrometry analysis, our group recently showed that MchA, McmL, MceC, on the hand, and MchS1, McmK, MceD, on the other hand, are required for siderophore-microcin post-translational modification, and that each group of genes displays the same function, i.e. enterobactin C-glucosylation and linearization, respectively (Vassiliadis et al., 2007; Vassiliadis et al., 2010). Similarly, MchC and MchD display $71 \%$ and $80 \%$ identity with MceJ and Mcel, respectively, and complement each other (Nolan and Walsh, 2008). Therefore, MchC and MceJ, on the one hand, as well as MchD and Mcel, on the other hand, most likely display the same function in O-glucosylation of the class II microcin C-terminal serine. Together with earlier heterologous functional complementation assays (Poey, Aspiroz and Laviña, 2006), this indicates that all genes involved in siderophore-microcin post-translational modification are probably interchangeable from one gene cluster to another.

Mccl47 and the putative MccG492 have been very recently described, and as a consequence, little is known on their maturation. However, Mccl47 structure and immunity genes are located within MccM/MccH47 gene cluster (Poey, Aspiroz and Laviña, 2006) while for MccG492 these genes are located within MccE492 gene cluster (Vassiliadis et al., 2010). Because MccM and MccH47 use the same genes for their post-translational modification, one can speculate that those same genes modify Mccl47 so that it carries a similar siderophore post-translational modification. Similarly, the genes required for MccE492 post-translational modification could modify MccG492.

A model is proposed for siderophore-microcin biosynthesis (Fig. 3) in which after biosynthesis, enterobactin is $C$-glucosylated by $\mathrm{MceC} / \mathrm{McmL} / \mathrm{MchA}$ and linearized by MceD/McmK/MchS1. The linearized glucosylated enterobactin (salmochelin) is then linked to the microcin precursor MceA (for MccE492), McmA (for MccM), MchB (for MccH47), MchS2 (for Mccl47) or MceL (for MccG492) through O-glycosylation. This biosynthetic step is catalyzed by McelJ or MchCD. Finally, cleavage of the leader peptide occurs simultaneous to the export by a dedicated ABC transporter encoded by the microcin gene cluster (see section 4.2.3.3.1). The existence of MccE492, MccM and MccH47 intermediates carrying a dimer and a monomer of glucosylated DHBS instead of the classical trimer (Vassiliadis et al., 2007; Vassiliadis et al., 2010) could result from the degradation of glucosylated enterobactin by MceD/McmK/MchS1, followed by linkage to the microcin precursor and cleavage of the leader peptide, as described above. 


\subsection{Maturation and export}

Analyses of class IIb microcin gene clusters led to several hypotheses on the cleavage of class IIb microcin leader peptide and associated export. The mechanism by which the microcins are maturated and exported is supposed to be the same for all class II microcins (see section 4.2.3.2.2). The ABC transporters of MccE492/MccG492 and MccH47/MccM/Mccl47 would be MceG and MchF, respectively. The accessory proteins of MccE492/MccG492 and MccH47/MccM/Mccl47 would be $\mathrm{MceH}$ and MchE, respectively. Finally, TolC would be the outer membrane component of all class Ilb microcin export machineries as shown for $\mathrm{MccH} 47$, whose antimicrobial activity is dependent on tolC (Gaggero et al., 1993). An additional inner membrane protein could also be required for class Ilb microcin export. Indeed, MccE492 export machinery seems to require MceF, a putative inner membrane protein that could interact with MceGH for processing or export (Lagos et al., 2001). Similarly, McmM, which displays $62 \%$ identity with MceF, could have the same function in $\mathrm{MccM} / \mathrm{MccH} 47 / \mathrm{Mccl} 47$ export.

\subsection{Recognition/uptake and mechanism of action}

Among class II microcins, the siderophore-microcins (class Ilb microcins) have been the best studied in terms of mechanism of action. While the mechanisms responsible for class Ilb microcin antibacterial activity are still incompletely understood, it is now established that their conserved Cterminal domain as well as their siderophore moiety are key determinants for their recognition/uptake at the outer membrane of enterobacteria.

\subsection{Recognition/uptake by the receptors for catecholate siderophores}

Spectra of antibacterial activity have been established very early for class Ilb microcins. However, as presented in the genetics section, class Ilb microcin-producing strains often express several class Ilb microcins. The recent progresses in the purification of microcins have rendered possible the determination of reliable but non-exhaustive activity spectra. Thus, using pure microcin preparations or preparations that contain one single microcin, MccE492 was shown to be active against strains of E. coli, Enterobacter cloacae, S. enterica, and K. pneumoniae, MccM was active against S. enterica and E. coli, while MccH47 would to be active against E. coli only (DestoumieuxGarzón et al., 2003; Thomas et al., 2004 Vassiliadis et al., 2010). 


\section{- Determinants of class Ilb microcin susceptibility in enterobacteria}

Two common features to all microcins are (i) an extremely potent activity, with minimum inhibitory concentrations (MICs) often below $0.1 \mu \mathrm{M}$ (Thomas et al., 2004; Destoumieux-Garzón et al., 2005), and (ii) a narrow spectrum of activity limited to few genera of enterobacteria, as illustrated above. The reason for that is a receptor-mediated recognition/uptake, which uses outer membrane proteins normally involved in nutrient uptake as receptors or docking molecules (Salomón and Farias, 1993; Laviña, Pugsley and Moreno, 1986; Pugsley, Moreno and de Lorenzo, 1986; Salomón and Farias, 1995; Trujillo, Rodríguez and Laviña, 2001). Those outer membrane proteins are also used by bacteriophages, antibiotics, and colicins for cell entry. They are considered an "Achilles' heel" for the bacterium.

\section{$\circ \quad$ The catecholate siderophore receptors FepA, Cir, Fiu and IroN}

Over the past years substantial knowledge has been acquired on microcin recognition showing that only enterobacteria that express catecholate siderophore receptors are susceptible to class Ilb microcins. In E. coli, class Ilb microcins parasitize the FepA, Cir and Fiu receptors involved in the uptake of catecholate siderophores (e.g. enterobactin) (Trujillo, Rodríguez and Laviña, 2001; Patzer et al., 2003; Thomas et al., 2004; Strahsburger et al., 2005). The requirement of MccE492 for catecholate siderophore receptors is illustrated by a shift from a potent activity against $E$. coli $\mathrm{H} 1443$ $(\mathrm{MIC}=40 \mathrm{nM})$ to a lack of activity against the isogenic fepA cir fiu triple mutant $(\mathrm{MIC}>10 \mu \mathrm{M})$ (Thomas et al., 2004). In Salmonella, class Ilb microcins would use IroN, FepA and Cir receptors (Patzer et al., 2003). FepA is by far the best studied of the four receptors. Its three-dimensional structure is composed of a $\beta$-barrel embedded in the outer membrane with an $\mathrm{N}$-terminal globular domain, either called the plug or the cork domain, folded inside the barrel (Buchanan et al., 1999). This domain spans most of the interior of the barrel and occludes it. The globular domain is connected to the $\beta$-barrel and to the external hydrophilic loops by numerous hydrogen bonds and salt bridges. The external loops contain the binding sites for iron-siderophore complexes (Annamalai et al., 2004). During the transport process, the globular domain of FepA would be expulsed from the $\beta$-barrel (Ma et al., 2007). Not only does FepA transport catecholate siderophores, but it also serves as a receptor/transporter for group B colicins and bacteriophages (Letellier and Santamaria, 2002; Rabsch et al., 2007). 


\section{$\circ$ The energy-transduction system TonB/ExbB/ExbD}

The catecholate siderophore receptors use the TonB-ExbB-ExbD inner membrane complex as an associated energy-transduction system for the active uptake of iron. This complex is responsible for the transduction of the proton-motive force energy from the inner membrane, where it is generated, to the outer membrane where it drives the active transport by high-affinity outer membrane transporters (Postle and Kadner, 2003). ExbB and ExbD were proposed to influence the dimerization of TonB although they would not be strictly required (Sauter, Howard and Braun, 2003), and TonBExbD interaction was recently shown to be dependent on the proton motive force (Ollis et al., 2009). TonB is required for the translocation across the outer membrane and the antibacterial activity of various microcins including class llb microcins (Braun et al., 2002; Pugsley et al., 1986; DestoumieuxGarzón et al., 2003; Thomas et al., 2004; Trujillo, Rodríguez and Laviña et al., 2001; Strahsburger et al., 2005; Vassiliadis et al., 2010). However, ExbB and ExbD are often dispensable, as evidenced for MccE492, MccM and MccH47 (Thomas et al., 2004; Vassiliadis et al., 2010). A similar ExbBindependent uptake has been reported for bacteriophage $\mathrm{H} 8$, which like class $\mathrm{Ilb}$ microcins and group B colicins is a FepA ligand. Interestingly, when both ExbBD and TolQR systems are impaired, E. coli becomes resistant to bacteriophage $\mathrm{H} 8$ infection (Rabsch et al., 2007). Similar functional complementation of ExbBD by TolQR may occur for the uptake of class Ilb microcins.

\section{- Determinants of class Ilb microcin recognition by catecholate siderophore receptors}

\section{- The salmochelin post-translational modification}

In a recent study, our group showed that the three class Ilb microcins on which structural data have been acquired (i.e. MccE492, MccM and MccH47) possess a similar post-translational modification (see structure section) when expressed in a favourable genetic background (Vassiliadis et al., 2010). Since this modification mimics the catecholate siderophore salmochelin and behaves as a siderophore (Thomas et al., 2004), it was hypothesized that it could play a major role in class Ilb microcin recognition. MccE492 is to date the only class $\mathrm{llb}$ microcin for which modified and unmodified forms have been separated and purified in sufficient amounts to measure minimal inhibitory concentrations (MICs). Interestingly, the activity of the unmodified MccE492 (u-MccE492) against $E$. coli $\mathrm{H} 1443$ is 4 to 8 -fold lower than that of the mature microcin (Thomas et al., 2004) indicating that the 
salmochelin post-translational modification significantly enhances MccE492 recognition at the outer membrane of $E$. coli. As shown recently, the same post-translational modification is observed in MccM, MccH47, and MccE492 (Vassiliadis et al., 2010), and is likely to occur in the recently discovered Mccl47 (Poey, Aspiroz and Laviña, 2006; Nolan and Walsh, 2008) and MccG492 (Vassiliadis et al., 2010). Therefore, as shown for MccE492, the salmochelin post-translational modification is expected to increase the antibacterial activity of all class Ilb microcins by mimicking the natural ligands of ironsiderophore receptors.

\section{$\circ \quad$ The serine-rich C-terminal region}

The unmodified MccE492 (u-MccE492) lacking the salmochelin post-translational modification was shown to be active against $E$. coli but, as the mature microcin, it was inactive against the isogenic fepA cir fiu triple mutant (Destoumieux-Garzón et al., 2006). This showed that molecular motives other than the sole post-translational modification are involved in MccE492 recognition by the catecholate siderophore receptors. Later, Bieler et al. showed that the serine-rich $C$-terminal sequence of MccE492 was essential for the activity of extracellular but not intracellular MccE492 (Bieler et al., 2006). Therefore, the $C$-terminal sequence is required for receptor recognition and/or translocation across the outer membrane. Because this C-terminal sequence is highly conserved among class Ilb microcins, being considered as a signature for the family, it is very likely that, as demonstrated for MccE492, all class Ilb microcins are recognized by catecholate-siderophore receptors through their serine-rich C-terminal sequence.

\subsection{Antibacterial activity of class Ilb microcins}

While class Ilb microcins exhibit a conserved C-terminal domain required for recognition, the $\mathrm{N}$ terminal domain is very diverse in terms of sequence (Table 1). Interestingly, the $\mathrm{N}$-terminal domains of class Ilb microcins display homologies with those of class Ila microcins. Therefore, class Illb Nterminal domains likely endow the antibacterial activity of the microcins. This is largely supported by the finding that a C-terminally truncated MccE492 remains active provided it is expressed intracellularly (Bieler et al., 2006). Among class Ilb microcins, only MccE492 and MccH47 have been investigated in terms of mechanism of action, certainly due to the recent identification of Mccl47, MccM and MccG492. Data on those last three microcins are limited to their recognition at the outer membrane of $E$. coli (see the above section). 
MccE492 membrane-disruption properties have been identified very early, well before the peptide was isolated and characterized. Thus, in vivo, culture supernatants containing MccE492 depolarised the inner membrane of E. coli (de Lorenzo and Pugsley, 1985). This was later confirmed with homogenous peptide preparations, showing that the MccE492 activity was energy- and TonBdependent (Destoumieux-Garzón et al., 2003; Destoumieux-Garzón et al., 2006). Interestingly, the damages to the inner membrane were not responsible by themselves for the lethal effect of neither uMccE492 nor MccE492, and did not result in cell lysis even at lethal concentrations (DestoumieuxGarzón et al., 2003; Destoumieux-Garzón et al., 2006). In vitro, the microcin was able to form ionchannels in planar lipid bilayers (Lagos et al., 1993). The u-MccE492 pore-forming activity could be observed at concentrations as low as $2 \times 10^{-10} \mathrm{M}$, and the insertion was shown to be voltageindependent (Destoumieux-Garzón et al., 2003). Altogether, this strongly suggests that the damages to $E$. coli inner membrane rely on a pore-forming activity.

Although the mechanistic details of MccE492 bactericidal activity are still incompletely understood, Bieler et al., evidenced that MccE492 targets the mannose permease, $\operatorname{man} Y$ and $\operatorname{man} Z$ being critical genes for MccE492 antibacterial activity against E. coli (Bieler et al., 2006). ManYZ is an inner membrane complex that functions together with the cytoplasmic ManX to form the mannose permease involved in the uptake of mannose and related hexoses (Erni et al., 1987; Williams et al., 1986). The manYZ mutants resistant to MccE492 were unable to metabolize mannose and became insensitive to the inner membrane depolarisation mediated by periplasmic MccE492 (Bieler et al., 2006). Therefore, the mannose permease is required for MccE492 antibacterial activity but may not be the only intracellular target of the microcin.

Unlike MccE492, MccH47 has never purified to homogeneity (Vassiliadis et al., 2010). Nonetheless, several studies by Laviña and collaborators have documented the mechanism of action of MccH47. The authors initially showed that the atp operon conferred susceptibility to $\mathrm{MccH} 47$ (Rodríguez, Gaggero and Laviña, 1999). Later, they found that $\mathrm{MccH} 47$ targets the $\mathrm{F}_{0} \mathrm{~F}_{1}$ ATP synthase, and particularly its $F_{0}$ membrane component, which serves as a proton channel, while the $F_{1}$ catalytic unit was dispensable (Trujillo, Rodríguez and Laviña, 2001; Rodríguez and Laviña, 2003). We recently found that contrary to MccE492, MccM and MccH47 are active against manXYZ mutants (Peduzzi and Vandervennet, unpublished results). Therefore the mannose permease is not required for all siderophore-microcins antibacterial activity. Consequently, while the conserved C-terminal 
sequence of class $\mathrm{Ilb}$ microcins is responsible for their recognition, this reinforces our hypothesis that their non-conserved $\mathrm{N}$-terminal sequence is responsible for the interference with specific cellular functions.

\section{Concluding remarks}

The recent progress on class II microcins gathered in this review have shown that their mechanism of action relies on the recognition by iron-siderophore receptors and that one subclass (class Ilb) uses molecular mimicry to improve recognition by iron receptors. Interestingly, the siderophores, which serve as precursors for the post-translational modification of class Ilb microcins, are recognized as important virulence factors involved in the battle for iron (Demir and Kaleli, 2004; Lawlor, O'Connor and Miller, 2007; Caza et al., 2008). Moreover, the siderophore-encoding gene clusters are usually located in genomic islands as also found for class Ilb microcins in E. coli Nissle 1917 (Grozdanov et al., 2004). Together with the modular structure of their gene cluster, this strongly suggests that class II microcins are subject to extensive horizontal gene transfer, in agreement with an important role in microbial competition and/or virulence. The recent studies on colicins, large antimicrobial proteins expressed by enterobacteria that use similar iron receptors for recognition, indicate that their expression favours bacterial persistence in the gastrointestinal tract (Kirkup and Riley, 2004; Gillor et al., 2009). Together with a recent study on uropathogenic class II microcins (Azpiroz, Poey and Laviña, 2009), this strongly supports the role of class II microcins in enterobacterial virulence and in vivo fitness.

\section{Acknowledgments}

We are grateful to Dominique Belin for the generous gifts of the E. coli manXYZ mutants. 


\section{References}

Andrews SC, Robinson AK, Rodriguez-Quinones F (2003) Bacterial iron homeostasis. FEMS Microbiol Rev 27: 215-237.

Annamalai R, Jin B, Cao Z, et al. (2004) Recognition of ferric catecholates by FepA. J Bacteriol 186: 3578-3589.

Azpiroz MF, Rodríguez E, Laviña M (2001) The structure, function, and origin of the microcin H47 ATP-binding cassette exporter indicate its relatedness to that of colicin V. Antimicrob Agents Chemother 45: 969-972.

Azpiroz MF, Laviña M (2004) Involvement of enterobactin synthesis pathway in production of microcin H47. Antimicrob Agents Chemother 48: 1235-1241.

Azpiroz MF, Poey ME, Laviña M (2009) Microcins and urovirulence in Escherichia coli. Microb Pathog 47: 274-280.

Bäumler AJ, Tsolis RM, van der Velden AW, et al. (1996) Identification of a new iron regulated locus of Salmonella typhi. Gene 183: 207-213.

Bäumler AJ, Norris TL, Lasco T, et al. (1998) IroN, a novel outer membrane siderophore receptor characteristic of Salmonella enterica. J Bacteriol 180: 1446-1453.

Benabdelhak H, Kiontke S, Horn C, et al. (2003) A specific interaction between the NBD of the ABCtransporter HlyB and a $C$-terminal fragment of its transport substrate haemolysin A. J Mol Biol 327: 1169-1179.

Bieler S, Silva F, Soto C, et al. (2006) Bactericidal activity of both secreted and nonsecreted microcin E492 requires the mannose permease. J Bacteriol 188: 7049-7061.

Bister B, Bischoff D, Nicholson GJ, et al. (2004) The structure of salmochelins: C-glucosylated enterobactins of Salmonella enterica. Biometals 17: 471-481.

Braun V, Patzer SI, Hantke K (2002) Ton-dependent colicins and microcins: modular design and evolution. Biochimie 84: 365-380.

Brickman TJ, Ozenberger BA, McIntosh MA (1990) Regulation of divergent transcription from the ironresponsive fepB-entC promoter-operator regions in Escherichia coli. J Mol Biol 212: 669-682.

Buchanan SK, Smith BS, Venkatramani L, et al. (1999) Crystal structure of the outer membrane active transporter FepA from Escherichia coli. Nat Struct Biol 6: 56-63.

Caza M, Lepine F, Milot S, et al. (2008) Specific roles of the iroBCDEN genes in virulence of an avian pathogenic Escherichia coli $\mathrm{O} 78$ strain and in production of salmochelins. Infect Immun 76: 35393549.

Chehade H, Braun V (1988) Iron-regulated synthesis and uptake of colicin V. FEMS Microbiol Lett 52: 177-182.

Cobbett CS, Delbridge ML (1987) Regulatory mutants of the aroF-tyrA operon of Escherichia coli K-12. J Bacteriol 169: 2500-2506.

Corsini G, Baeza M, Monasterio O, et al. (2002) The expression of genes involved in microcin maturation regulates the production of active microcin E492. Biochimie 84: 539-544.

Crosa JH, Walsh CT (2002) Genetics and assembly line enzymology of siderophore biosynthesis in bacteria. Microbiol Mol Biol Rev 66: 223-249. 
Davidson AL, Maloney PC (2007) ABC transporters: how small machines do a big job. Trends Microbiol 15: 448-455.

de Keyzer J, van der Does C, Driessen AJ (2003) The bacterial translocase: a dynamic protein channel complex. Cell Mol Life Sci 60: 2034-2052.

de Lorenzo V (1984) Isolation and characterization of microcin E492 from Klebsiella pneumoniae. Arch Microbiol 139: 72-75.

de Lorenzo V, Pugsley AP (1985) Microcin E492, a low-molecular-weight peptide antibiotic which causes depolarization of the Escherichia coli cytoplasmic membrane. Antimicrob Agents Chemother 27: 666-669.

Demir M, Kaleli I (2004) Production by Escherichia coli isolates of siderophore and other virulence factors and their pathogenic role in a cutaneous infection model. Clin Microbiol Infect 10: 10111014.

Destoumieux-Garzón D, Thomas X, Santamaria M, et al. (2003) Microcin E492 antibacterial activity: evidence for a TonB-dependent inner membrane permeabilization on Escherichia coli. Mol Microbiol 49: 1031-1041.

Destoumieux-Garzón D, Duquesne S, Peduzzi J, et al. (2005) The iron-siderophore transporter FhuA is the receptor for the antimicrobial peptide microcin J25: role of the microcin Val11-Pro16 betahairpin region in the recognition mechanism. Biochem J 389: 869-876.

Destoumieux-Garzón D, Peduzzi J, Thomas X, et al. (2006) Parasitism of iron-siderophore receptors of Escherichia coli by the siderophore-peptide microcin E492m and its unmodified counterpart. Biometals 19: 181-191.

Dezfulian H, Tremblay D, Harel J (2004) Molecular characterization of extraintestinal pathogenic Escherichia coli (ExPEC) pathogenicity islands in F165-positive E. coli strain from a diseased animal. FEMS Microbiol Lett 238: 321-332.

Dobrindt U, Blum-Oehler G, Hartsch T, et al. (2001) S-Fimbria-encoding determinant sfa(I) is located on pathogenicity island III(536) of uropathogenic Escherichia coli strain 536. Infect Immun 69: 4248-4256.

Duquesne S, Destoumieux-Garzón D, Peduzzi J, et al. (2007) Microcins, gene-encoded antibacterial peptides from enterobacteria. Nat Prod Rep 24: 708-734.

Ecker DJ, Matzanke BF, Raymond KN (1986) Recognition and transport of ferric enterobactin in Escherichia coli. J Bacteriol167: 666-673.

Erni B, Zanolari B, Kocher HP (1987) The mannose permease of Escherichia coli consists of three different proteins. Amino acid sequence and function in sugar transport, sugar phosphorylation, and penetration of phage lambda DNA. J Biol Chem 262: 5238-5247.

Fiedler HP, Krastel P, Müller J, et al. (2001) Enterobactin: the characteristic catecholate siderophore of Enterobacteriaceae is produced by Streptomyces species. FEMS Microbiol Lett 196: 147-151.

Fischbach MA, Lin H, Liu DR, et al (2005) In vitro characterization of IroB, a pathogen-associated Cglycosyltransferase. Proc Natl Acad Sci (USA) 102: 571-576.

Franke CM, Leenhouts KJ, Haandrikman AJ, et al. (1996) Topology of LcnD, a protein implicated in the transport of bacteriocins from Lactococcus lactis. J Bacteriol 178: 1766-1769. 
Franke CM, Tiemersma J, Venema G, et al. (1999) Membrane topology of the lactococcal bacteriocin ATP-binding cassette transporter protein LcnC. Involvement of $\mathrm{LcnC}$ in lactococcin a maturation. J Biol Chem 274: 8484-8490.

Fredericq P, Joiris E, Betz-Barreau M, et al. (1949) Recherche des gènes producteurs de colicines dans les selles de malades atteints de fièvre paratyphoïde B. C R Soc Biol 143: 556-559.

Gaggero C, Moreno F, Laviña M (1993) Genetic analysis of microcin H47 antibiotic system. J Bacteriol 175: 5420-5427.

Gaillard-Gendron S, Vignon D, Cottenceau G, et al. (2000) Isolation, purification and partial amino acid sequence of a highly hydrophobic new microcin named microcin $\mathrm{L}$ produced by Escherichia coli. FEMS Microbiol Lett 193: 95-98.

Gehring AM, Bradley KA, Walsh CT (1997) Enterobactin biosynthesis in Escherichia coli: isochorismate lyase (EntB) is a bifunctional enzyme that is phosphopantetheinylated by EntD and then acylated by EntE using ATP and 2,3-dihydroxybenzoate. Biochemistry 36: 8495-8503.

Gehring AM, Mori I, Walsh CT (1998) Reconstitution and characterization of the Escherichia coli enterobactin synthetase from EntB, EntE, and EntF. Biochemistry 37: 2648-2659.

Gerard F, Pradel N, Wu LF (2005) Bactericidal activity of colicin V is mediated by an inner membrane protein, SdaC, of Escherichia coli. J Bacteriol 187: 1945-1950.

Gillor O, Giladi I, Riley MA (2009) Persistence of colicinogenic Escherichia coli in the mouse gastrointestinal tract. BMC Microbiol 9: 165.

Gilson L, Mahanty HK, Kolter R (1987) Four plasmid genes are required for colicin V synthesis, export, and immunity. J Bacteriol 169: 2466-2470.

Gilson L, Mahanty HK, Kolter R (1990) Genetic analysis of an MDR-like export system: the secretion of colicin V. Embo J 9: 3875-3884.

Gratia A (1925) Sur un remarquable exemple d'antagonisme entre deux souches de colibacille. C R Soc Biol 93: 1041-1042.

Grove CL, Gunsalus RP (1987) Regulation of the aroH operon of Escherichia coli by the tryptophan repressor. J Bacteriol 169: 2158-2164.

Grozdanov L, Raasch C, Schulze J, et al. (2004) Analysis of the genome structure of the nonpathogenic probiotic Escherichia coli strain Nissle 1917. J Bacteriol 186: 5432-5441.

Guo X, Harrison RW, Tai PC (2006) Nucleotide-dependent dimerization of the C-terminal domain of the ABC transporter CvaB in colicin V secretion. J Bacteriol 188: 2383-2391.

Hantke K, Nicholson G, Rabsch W, et al. (2003) Salmochelins, siderophores of Salmonella enterica and uropathogenic Escherichia coli strains, are recognized by the outer membrane receptor IroN. Proc Natl Acad Sci (USA) 100: 3677-3682.

Håvarstein LS, Holo H, Nes IF (1994) The leader peptide of colicin V shares consensus sequences with leader peptides that are common among peptide bacteriocins produced by gram-positive bacteria. Microbiology 140: 2383-2389.

Håvarstein LS, Diep DB, Nes IF (1995) A family of bacteriocin ABC transporters carry out proteolytic processing of their substrates concomitant with export. Mol Microbiol 16: 229-240. 
Heatwole VM, Somerville RL (1992) Synergism between the Trp repressor and Tyr repressor in repression of the aroL promoter of Escherichia coli K-12. J Bacteriol 174: 331-335.

Hollenstein K, Dawson RJ, Locher KP (2007) Structure and mechanism of ABC transporter proteins. Curr Opin Struct Biol 17: 412-418.

Kirkup BC, Riley MA (2004) Antibiotic-mediated antagonism leads to a bacterial game of rock-paperscissors in vivo. Nature 428: 412-414.

Koronakis V, Sharff A, Koronakis E, et al. (2000) Crystal structure of the bacterial membrane protein TolC central to multidrug efflux and protein export. Nature 405: 914-919.

Koronakis V, Eswaran J, Hughes C (2004) Structure and function of TolC: the bacterial exit duct for proteins and drugs. Annu Rev Biochem 73: 467-489.

Lagos R, Wilkens M, Vergara C, et al. (1993) Microcin E492 forms ion channels in phospholipid bilayer membrane. FEBS Lett 321: 145-148.

Lagos R, Villanueva JE, Monasterio O (1999) Identification and properties of the genes encoding microcin E492 and its immunity protein. J Bacteriol 181: 212-217.

Lagos R, Baeza M, Corsini G, et al. (2001) Structure, organization and characterization of the gene cluster involved in the production of microcin E492, a channel-forming bacteriocin. Mol Microbiol 42: 229-243.

Lambalot RH, Gehring AM, Flugel RS, et al. (1996) A new enzyme superfamily - the phosphopantetheinyl transferases. Chem Biol 3: 923-936.

Laviña M, Pugsley AP, Moreno F (1986) Identification, mapping, cloning and characterization of a gene (sbmA) required for microcin B17 action on Escherichia coli K12. J Gen Microbiol 132: 16851693.

Laviña M, Gaggero C, Moreno F (1990) Microcin H47, a chromosome-encoded microcin antibiotic of Escherichia coli. J Bacteriol 172: 6585-6588.

Lawlor MS, O'Connor C, Miller VL (2007) Yersiniabactin is a virulence factor for Klebsiella pneumoniae during pulmonary infection. Infect Immun 75: 1463-1472.

Letellier L, Santamaria M (2002) The biochemical and physiological characteristics of surface receptors of gram negative bacteria. Mini Rev Med Chem 2: 343-351.

Lin H, Fischbach MA, Liu DR, et al. (2005) In vitro characterization of salmochelin and enterobactin trilactone hydrolases IroD, IroE, and Fes. J Am Chem Soc 127: 11075-11084.

Ma L, Kaserer W, Annamalai R, et al. (2007) Evidence of ball-and-chain transport of ferric enterobactin through FepA. J Biol Chem 282: 397-406.

Mercado G, Tello M, Marin M, et al (2008) The production in vivo of microcin E492 with antibacterial activity depends on salmochelin and EntF. J Bacteriol 190: 5464-5471.

Miethke M, Marahiel MA (2007) Siderophore-based iron acquisition and pathogen control. Microbiol Mol Biol Rev 71: 413-451.

Moussatova A, Kandt C, O'Mara ML, et al. (2008) ATP-binding cassette transporters in Escherichia coli. Biochim Biophys Acta 1778: 1757-1771.

Neilands JB (1995) Siderophores: structure and function of microbial iron transport compounds. J Biol Chem 270: 26723-26726. 
Nissle A (1925) Weiteres über Grundlagen und Praxis der Mutaflorbehandlung. Dtsch Med Wochenschr 44: 1809-1813.

Nolan EM, Fischbach MA, Koglin A, et al. (2007) Biosynthetic Tailoring of Microcin E492m: Posttranslational Modification Affords an Antibacterial Siderophore-Peptide Conjugate. J Am Chem Soc. 129: 14336-14347.

Nolan EM, Walsh CT (2008) Investigations of the McelJ-catalyzed posttranslational modification of the microcin E492 C-terminus: linkage of ribosomal and nonribosomal peptides to form "trojan horse" antibiotics. Biochemistry 47: 9289-9299.

O'Brien IG, Cox GB, Gibson F (1970) Biologically active compounds containing 2,3-dihydroxybenzoic acid and serine formed by Escherichia coli. Biochim Biophys Acta 201: 453-460.

O'Brien GJ, Mahanty HK (1994) Colicin 24, a new plasmid-borne colicin from a uropathogenic strain of Escherichia coli. Plasmid 31: 288-296.

Ollis AA, Manning M, Held KG, et al. (2009) Cytoplasmic membrane protonmotive force energizes periplasmic interactions between ExbD and TonB. Mol Microbiol 73: 466-481.

Patzer SI, Baquero MR, Bravo D, et al. (2003) The colicin G, H and X determinants encode microcins $\mathrm{M}$ and $\mathrm{H} 47$, which might utilize the catecholate siderophore receptors FepA, Cir, Fiu and IroN. Microbiology 149: 2557-2570.

Payne SM, Niesel DW, Peixotto SS, et al. (1983) Expression of hydroxamate and phenolate siderophores by Shigella flexneri. J Bacteriol 155: 949-955.

Podschun R, Fischer A, Ullmann U (1992) Siderophore production of Klebsiella species isolated from different sources. Zentralbl Bakteriol 276: 481-486.

Poey ME, Azpiroz MF, Laviña M (2006) Comparative analysis of chromosome-encoded microcins. Antimicrob Agents Chemother 50: 1411-1418.

Pollack JR, Neilands JB (1970) Enterobactin, an iron transport compound from Salmonella Thyphimurium. Biochem Biophys Res Commun 38: 989-992.

Pons AM, Zorn N, Vignon D, et al. (2002) Microcin E492 is an unmodified peptide related in structure to colicin V. Antimicrob Agents Chemother 46: 229-230.

Pons AM, Delalande F, Duarte M, et al. (2004) Genetic analysis and complete primary structure of microcin L. Antimicrob Agents Chemother 48: 505-513.

Postle K, Kadner RJ (2003) Touch and go: tying TonB to transport. Mol Microbiol 49: 869-882.

Pugsley AP, Moreno F, de Lorenzo V (1986) Microcin-E492-insensitive mutants of Escherichia coli K12. J Gen Microbiol 132: 3253-3259.

Rabsch W, Ma L, Wiley G, et al. (2007) FepA- and TonB-dependent bacteriophage H8: receptor binding and genomic sequence. J Bacteriol 189: 5658-5674.

Raymond KN, Dertz EA, Kim SS (2003) Enterobactin: an archetype for microbial iron transport. Proc Natl Acad Sci (USA) 100: 3584-3588.

Reichert J, Sakaitani M, Walsh CT (1992) Characterization of EntF as a serine-activating enzyme. Protein Sci 1: 549-556.

Rodríguez E, Laviña M (1998) Genetic analysis of microcin H47 immunity. Can J Microbiol 44: 692697. 
Rodríguez E, Gaggero C, Laviña M (1999) The structural gene for microcin H47 encodes a peptide precursor with antibiotic activity. Antimicrob Agents Chemother 43: 2176-2182.

Rodríguez E, Laviña M (2003) The proton channel is the minimal structure of ATP synthase necessary and sufficient for microcin h47 antibiotic action. Antimicrob Agents Chemother 47: 181-187.

Sablé S, Duarte M, Bravo D, et al. (2003) Wild-type Escherichia coli producing microcins B17, D93, J25, and L; cloning of genes for microcin L production and immunity. Can J Microbiol 49: 357-361.

Sakaitani M, Rusnak F, Quinn NR, et al. (1990) Mechanistic studies on trans-2,3-dihydro-2,3dihydroxybenzoate dehydrogenase (Ent $A$ ) in the biosynthesis of the iron chelator enterobactin. Biochemistry 29: 6789-6798.

Salomón RA, Farias RN (1993) The FhuA protein is involved in microcin 25 uptake. J Bacteriol 175: 7741-7742.

Salomón RA, Farias RN (1995) The peptide antibiotic microcin 25 is imported through the TonB pathway and the SbmA protein. J Bacteriol 177: 3323-3325.

Sauter A, Howard SP, Braun V (2003) In vivo evidence for TonB dimerization. J Bacteriol 185: 57475754 .

Shaw-Reid CA, Kelleher NL, Losey HC, et al. (1999) Assembly line enzymology by multimodular nonribosomal peptide synthetases: the thioesterase domain of E. coli EntF catalyzes both elongation and cyclolactonization. Chem Biol 6: 385-400.

Skvirsky RC, Reginald S, Shen X (1995) Topology analysis of the colicin V export protein CvaA in Escherichia coli. J Bacteriol 177: 6153-6159.

Strahsburger E, Baeza M, Monasterio O, et al. (2005) Cooperative uptake of microcin E492 by receptors FepA, Fiu, and Cir and inhibition by the siderophore enterochelin and its dimeric and trimeric hydrolysis products. Antimicrob Agents Chemother 49: 3083-3086.

Thomas X, Destoumieux-Garzón D, Péduzzi J, et al. (2004) Siderophore peptide, a new type of posttranslationally modified antibacterial peptide with potent activity. J Biol Chem 279: 28233-28242.

Trujillo M, Rodríguez E, Laviña M (2001) ATP synthase is necessary for microcin $\mathrm{H} 47$ antibiotic action. Antimicrob Agents Chemother 45: 3128-3131.

Vassiliadis G, Peduzzi J, Zirah S, et al. (2007) Insight into siderophore-carrying Peptide biosynthesis: enterobactin is a precursor for microcin E492 posttranslational modification. Antimicrob Agents Chemother 51: 3546-3553.

Vassiliadis G, Destoumieux-Garzón D, Lombard C, et al. (2010) Isolation and characterization of two members of the siderophore-microcin family, microcins $\mathrm{M}$ and H47. Antimicrob Agents Chemother 54: 288-297.

Walsh CT, Liu J, Rusnak F, et al. (1990) Molecular studies on enzymes in chorismate metabolism and the enterobactin biosynthetic pathway. Chem Rev 90: 1105-1129.

Waters VL, Crosa JH (1991) Colicin V virulence plasmids. Microbiol Rev 55: 437-450.

Welch RA, Burland V, Plunkett G, 3rd, et al. (2002) Extensive mosaic structure revealed by the complete genome sequence of uropathogenic Escherichia coli. Proc Natl Acad Sci (USA) 99: 17020-17024. 
Wilkens M, Villanueva JE, Cofre J, et al. (1997) Cloning and expression in Escherichia coli of genetic determinants for production of and immunity to microcin E492 from Klebsiella pneumoniae. $\mathrm{J}$ Bacteriol 179: 4789-4794.

Williams N, Fox DK, Shea C, et al. (1986) Pel, the protein that permits lambda DNA penetration of Escherichia coli, is encoded by a gene in ptsM and is required for mannose utilization by the phosphotransferase system. Proc Natl Acad Sci (USA) 83: 8934-8938.

Wooley RE, Gibbs PS, Shotts EB, Jr. (1999) Inhibition of Salmonella typhimurium in the chicken intestinal tract by a transformed avirulent avian Escherichia coli. Avian Dis 43: 245-250.

Wu TK, Huang CY, Ko CY, et al. (2004) Purification, tandem mass characterization, and inhibition studies of oxidosqualene-lanosterol cyclase enzyme from bovine liver. Arch Biochem Biophys 421: 42-53.

Yang CC, Konisky J (1984) Colicin V-treated Escherichia coli does not generate membrane potential. J Bacteriol 158: 757-759.

Zhong X, Kolter R, Tai PC (1996) Processing of colicin V-1, a secretable marker protein of a bacterial ATP binding cassette export system, requires membrane integrity, energy, and cytosolic factors. J Biol Chem 271: 28057-28063.

Zhu M, Valdebenito M, Winkelmann G, et al. (2005) Functions of the siderophore esterases IroD and IroE in iron-salmochelin utilization. Microbiology 151: 2363-2372. 


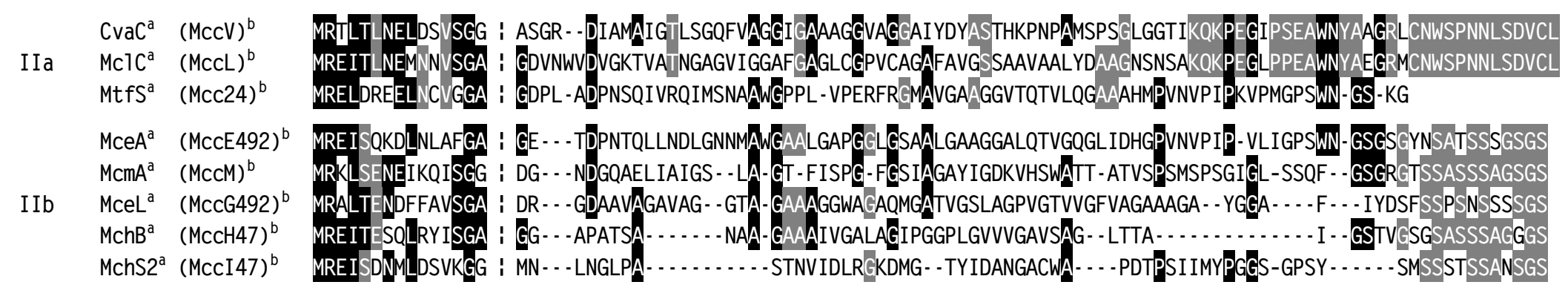

a Name of the microcin precursors. ${ }^{b}$ Name of the mature microcins (known or putative).

A vertical dash indicates the known or putative cleavage site of microcin precursors. Dashes in the amino acid sequence indicate gaps. Amino acids conserved in at least $50 \%$ of both class IIa and IIb sequences are highlighted in black. Amino acids conserved in at least $66 \%$ of class IIa sequences or $60 \%$ of class IIb sequences are highlighted in grey. 


\section{Captions to figures}

Fig. 1 Genetic organization of class lla microcin biosynthetic gene clusters. A colour code identifies genes with similar functions. Genes encoding microcin precursors are shown in white. Genes required for self-immunity and microcin export are shown in black and grey, respectively. Promoters are indicated by flags. The truncated gene in MccL gene cluster is crossed through.

Fig. 2 Genetic organization of class Ilb microcin biosynthetic gene clusters. A colour code identifies genes with similar functions. Genes encoding microcin precursors are shown in white. Genes required for self-immunity and microcin export are shown in black and grey, respectively. Genes involved in the post-translational modification are indicated with right- and left-inclined hatching. Genes encoding transposases and proteins of unknown function are indicated with horizontal hatching and vertical hatching, respectively. Truncated genes are crossed through.

Fig. 3 Model for the class Ilb biosynthesis. u-microcin corresponds to unmodified microcin while umicrocin-Glc-DHBS and u-microcin-Glc-DHBS ${ }_{2}$ correspond to microcin intermediate forms. Ent, Glc and DHBS stand for enterobactin, glucose, and $\mathrm{N}$-(2,3 dihydroxybenzoyl)-L-serine, respectively. 


\section{$\mathrm{MccV}$}

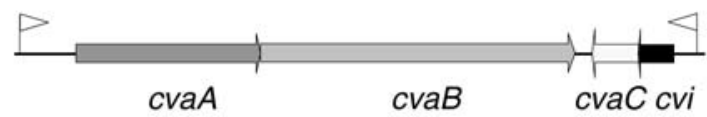

MccL

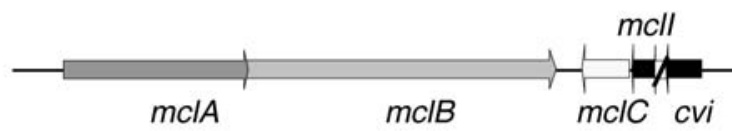

Mcc24

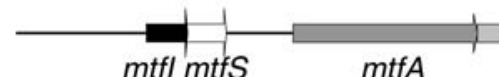




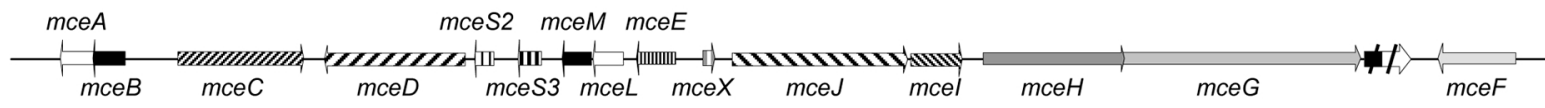

\section{- E. coli H47 (MccH47/147)}

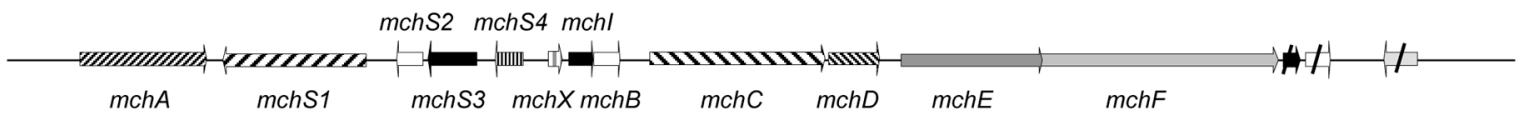

- E. coli CA46 (MccM/H47//47)

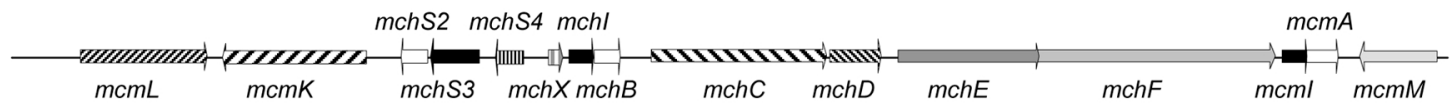

\section{- E. coli CA58 (MccM/H47)}

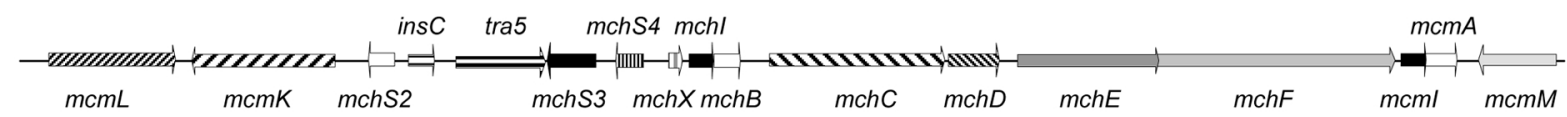

- E. coli Nissle 1917 (MccM/H47)

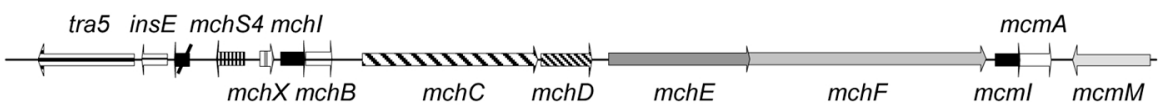


